\title{
Attentional Networks in Co-occurring Generalized Anxiety Disorder and Major \\ Depression Disorder: Towards a Staging Approach to the Executive Control Deficits
}

Charlotte Coussement ${ }^{1,2}$, Xavier De Longueville ${ }^{3}$, and Alexandre Heeren ${ }^{1,4}$

${ }^{1}$ Psychological Sciences Research Institute, UCLouvain, Belgium

${ }^{2}$ Le Beau Vallon - Psychiatric Hospital, Belgium

${ }^{3}$ Department of Adult Psychiatry, Cliniques Universitaires Saint-Luc, Brussels, Belgium

${ }^{4}$ Institute of Neuroscience, UCLouvain, Belgium

\section{Corresponding author:}

Prof. Alexandre Heeren, Ph.D.

Psychological Sciences Research Institute \& Instiute of Neuroscience,

Université Catholique de Louvain (UCLouvain)

Place du Cardinal Mercier, 10, B-1348, Louvain-la-Neuve, Belgium.

Email: alexandre.heeren@uclouvain.be 


\begin{abstract}
Introduction. Major Depression Disorder (MDD) and Generalized Anxiety Disorder (GAD) often co-occur, but the neurocognitive mechanisms of this co-occurrence remain unknown. Prominent views have pointed to attentional processes as potent mechanisms at play in MDD and GAD, respectively. Yet uncertainty remains regarding the very nature of attentional impairments in patients with co-occurring MDD and GAD.
\end{abstract}

Methods. Inspired by contemporary models of attentional networks, we compared the three main attentional networks, namely the orienting, alerting, and executive networks of the Attention Network Task, in four groups of patients with, respectively, co-occurring DSM-5 MDD and GAD $(n=30)$, DSM-5 MDD only $(n=30)$, DSM-5 GAD only $(n=30)$, or free from any DSM-5 diagnosis $(n=30)$. To capture the multivariate nature of our data, we examined between-group differences in the attentional networks through a multivariate analysis of variance.

Results. Patients with co-occurring MDD and GAD exhibited more severe impairments in the executive control network than those with only one of the disorders. Although patients with MDD or GAD solely did not differ in attentional impairments, both groups showed significantly more impairments in the executive control network than those without either MDD and GAD (all Bonferonni-corrected post-hoc $p \mathrm{~s}<.05$ ).

Conclusions. Our findings align with a longstanding staging approach to comorbidity whereby, via synergistic effects, co-occurring disorders may produce more damages than the sum of each disorder. Here, for the first time, we extended this approach to the executive network of attention in the context of the co-occurrence between MDD and GAD.

Keywords: Attentional Networks, Executive control, Major Depression Disorder, Generalized Anxiety Disorder, Comorbidity 


\section{Introduction}

With lifetime comorbidity rates of nearly one-half [1,2], major depressive disorder (MDD) and generalized anxiety disorder (GAD) often co-occur [3]. For instance, in the general population, $51 \%$ of people qualifying for the diagnosis of MDD also meet, in the same year, the diagnostic criteria of an anxiety disorder, while only $11.8 \%$ of the people without MDD do [4]. Likewise, studies conducted in the general population indicated that individuals with GAD have a higher risk for developing an MDD in the years following the onset of GAD [5], particularly within the first year succeeding the onset of GAD [6].

The comorbidity between GAD and MDD has severe clinical implications. First, it increases the risk of persistence and recurrence of both disorders [7-9] as well as the likelihood of developing somatic complaints, like cardiovascular problems, ulcers, or migraines [10]. Second, patients with co-occurring GAD and MDD have a higher risk of attempting suicide than patients qualifying for only one of these disorders [7-11]. Finally, patients with cooccurring MDD and GAD benefit less from pharmacological or psychological treatments [1214] and maintain fewer therapeutic gains over time [15,16]. Notably, some specific MDD symptoms, such as concentration and attention problems, may interfere with pharmacological and psychological treatments. In light of these adverse consequences, clarifying the mechanisms of the co-occurrence between GAD and MDD appears as a critical and timely step to usher the field forward.

Research tagging attention control-i.e., the ability to voluntarily regulate the allocation of attentional resources $[17,18]$ — as a potential neurocognitive mechanism of GAD and MDD has increasingly gained traction in the last decade [17, 19-21]. Moreover, the recent Research Domain Criteria (RDoC) initiative has placed attention control in one of its five central dimensions for transdiagnostic mental health research $[22,23]$. Note that this view should not 
come as a surprise as the implication of attention in mood and anxiety has been discussed by scholars for a long time [24].

Yet, although extensive empirical research has been done on investigating the attentional impairments in patients with either GAD or MDD [19, 25-28], attentional impairments in patients with co-occurring GAD and MDD have seldom been investigated. Moreover, research has so far led to mixed findings and inconclusive outcomes. Notably, two studies have thoroughly compared MDD patients with and without comorbid anxiety disorders on a broad battery of neuropsychological tests $[29,30]$. Both studies reported that depressed patients with comorbid anxiety showed impaired performance on tasks assessing attention and executive control. In contrast, they found that depressed patients without comorbid anxiety did not exhibit any impairment, thus pointing to a specific debilitating role of the comorbid anxiety in the impairment of attention control. However, other studies [31,32] did not find any difference between MDD patients with and without comorbid anxiety disorders in tasks measuring attention or executive functions, challenging the claim that comorbid anxiety might damage attention control.

However, in most of these studies, the very notion of "comorbid anxiety" referred to distinct clinical phenomena, with each of these studies relying on a different conceptualization. For instance, in some studies [30], comorbid anxiety denoted a high score on a scale assessing various features of anxiety. In contrast, in other research, comorbid anxiety signified the presence of at least one concurrent anxiety disorder - though often more than one comorbid disorder [29]. Furthermore, although all previous studies contrasted the attentional abilities of MDD patients with comorbid anxiety to those of depressed patients without anxiety, none of these studies included anxious patients without depression. As a result, because prior research only relied upon research designs including at most three groups (i.e., patients with MDD and co-occurring anxiety disorders; patients with MDD only; healthy comparison group), 
uncertainty remains regarding the respective influence of GAD only (devoid of MDD) on the ANT performances observed in people with co-occurring MDD and GAD [29-30]. This methodological flaw is especially troubling given the well-documented literature on the impacts of anxiety on attentional abilities (for a meta-analysis, see [33-35]).

Altogether, these limitations seriously impede the inference and generalization that one can make from these previous studies. As such, the very contribution of depression versus anxiety in impaired attention control among patients with co-occurring MDD and GAD remains unclear. To consider GAD's specific role and help the field move forward, the present research relies on patients with MDD solely, GAD solely, or co-occurring GAD and MDD, compared to healthy volunteers.

Another critical issue of prior research on the co-occurrence between MDD and GAD pertains to the experimental tasks assessing attention control. Indeed, previous studies relied on various neuropsychological tasks, simultaneously exploring several distinct attentional functions, ranging from lower levels of the attention systems (e.g., selective attention) to topdown executive attention control. However, most of these tasks were devoid of strong theoretical foundations, leading to the use of tasks lacking a sound theoretical conceptualization of attention and its subcomponents. As such, most of the previous research on the co-occurrence between MDD and GAD did not examine attentional impairments through the lens of different theory-driven approaches to the attentional system; therefore, thwarting theoretical refinement and ultimately blocking scientific and clinical progress [36].

Thus, a decisive and timely step to help usher forward research into the co-occurrence between depression and anxiety involves exploring attentional impairments through the lens of a sound integrative model of the attention system and its subcomponents. According to the attention-network perspective of attention (e.g., [37-41]), the attention systems can be conceptualized as a multifaceted construct including three neuroanatomically (but overlapping 
brain regions) and functionally distinct attentional networks (for a review, see [40]). These are the alerting network (i.e., maintenance of alertness), the orienting network (i.e., selective engagement and disengagement with certain stimuli rather than others), and the executive network of attention (i.e., top-down control of attention exemplified by the maintenance of attention on certain stimuli and resisting distraction by other stimuli) $[38,39]$.

To assess these three networks, scholars have devised the Attention Network Test (ANT) [37], which combines attentional and spatial cues with a flanker task [42, 43] (i.e., a central imperative stimulus is flanked by distractors that indicate either the same or opposite response to the imperative stimulus). In this task, a spatial cue is presented onscreen for each trial, followed by an array of five arrows located at either the top or the bottom of the computer screen (see Figure 1). The participant is asked to indicate the direction of the central arrow as rapidly as possible. The cue that precedes the arrows can be non-existent (i.e., no cue), a center cue, a double cue (one presented at each of the two possible target locations), or a spatial cue that deterministically indicates the upcoming target location. The ANT has been developed to reliably evaluate three attentional networks: the alerting, orienting, and executive control network. The alerting network contrasts performance with and without cues, the orienting network distinguishes performance on the task with or without a reliable spatial cue, and the executive network (conflict) is measured by interference from flankers.

Given the theoretical and metric foundations of the ANT, many scholars have capitalized on this task to probe the nature of the attentional impairments in patients with psychiatric [44-49] and neurological disorders [50-52]. Moreover, concerning MDD and GAD, the ANT has already been adopted to examine the distinct components of the attentional networks involved in anxiety disorders, with studies pointing to impairment in the orienting network in social anxiety disorder $[49,52,53]$ and an impaired executive control network in patients with various other anxiety disorders [54]-i.e., either panic disorder, GAD, obsessive- 
compulsive disorder, or posttraumatic stress disorder. Likewise, the ANT has also been implemented in mood disorder research, with several studies reporting impairments in the executive network in patients with MDD [49] and bipolar disorders $[55,56]$.

Despite the ability of the ANT to grant new insights on the attentional impairments in patients with co-occurring GAD and MDD [17,19,20,57], this task has curiously not yet been adopted to compare patients with co-occurring MDD and GAD. Thus, the present study aimed at probing, for the first time, the potential impairments in the three attentional networks among patients with MDD solely, GAD solely, or co-occurring GAD and MDD, as compared to healthy volunteers.

\section{Method}

\subsection{Ethical statement}

Participants received complete details about the aims of the study and the procedure and we obtained written informed consent from each of them. The study received the approval of the Institutional Review Board B403201629865 and was carried out under the Helsinki Declaration.

\subsection{Participants}

The sample consisted of 120 participants (73\% women). Among them, 30 patients qualified for the DSM-5 diagnosis of MDD without GAD (hereafter MDD), 30 for GAD without MDD (hereafter GAD), 30 for both diagnoses (hereafter MDD/GAD), and 30 healthy comparison participants, qualifying neither for MDD nor GAD (hereafter, HC). Patients were recruited from the "Beau Vallon" Psychiatric Hospital (Belgium) and local community listservs for clinical psychology. Exclusion criteria were: (a) the presence of other psychiatric disorders, (b) current or past heart or neurological problems, (c) pregnancy at the time of the testing, (d) impaired visual functioning, and (e) insufficient knowledge of the French language. These criteria were checked through a medical interview and an evaluation of DSM-5 mental disorders 
[58]. A certified CBT therapist completed all the interviews. $\mathrm{HC}$ were recruited through the institutional volunteer pool. Their absence of current and past psychiatric symptomatology was checked using the DSM-5 criteria interview. Participants'characteristics are in Table 1.

\subsection{Measures}

\subsubsection{Questionnaires.}

All participants completed the Beck Depression Inventory (BDI-II) [59] and the Generalized Anxiety Disorder Scale (GAD-7) [60]. The BDI-II is a self-reported questionnaire measuring depression symptoms via 21 items. The GAD-7 is a self-reported questionnaire measuring GAD symptoms via 7 items. We used the validated French versions of these scales $[59,61]$. Their internal reliability was high in the current sample, with Cronbach's alphas of .94 and .91, repectively, for the BDI-II and the GAD-7.

\subsubsection{Experimental measure.}

We assessed the three independent attentional networks via the ANT [37]. In this task, participants were instructed to determine, by clicking as quickly and accurately as possible on the corresponding button (i.e., left or right), the direction of a target, a central arrow flanked by four distractors that indicate either the same or opposite response and this array of arrows (or dashes, see below) was projected either at the top or the bottom of the screen. The target remained on screen until a response was detected (or for $1700 \mathrm{~ms}$ if no response occurred). A schematic representation of the ANT appears in Figure 1.

The cue that preceded the arrays of arrows can be non-existent (i.e., no cue), a center cue (i.e., an asterisk replacing the fixation cross), a double cue (two asterisks, each presented of one of the two possible target locations), or a spatial cue (an asterisk positioned above or below the fixation cross and deterministically indicating the upcoming target location). In 
addition to the target, the task also involved flankers appearing horizontally on each side of the target. As shown in Figure 1, there were three possible flanker types: congruent (i.e., two arrows pointing in the same direction as the target), incongruent (i.e., two arrows pointing in the direction opposite to the target), and neutral (i.e., two dashes).

Given the potential combination of the four types of cues, the three types of flankers, and the two directions of the target arrow (see Figure 1), there were 48 possible trials. The experiment included three blocks of 96 trials each (presenting twice the 48 possible trials), resulting in 288 trials presented in random order. There were short breaks between blocks. For each trial, RT (in ms) and accuracy were recorded.

\subsection{Procedure}

Participants completed the task individually in a quiet, dimly lit room and were sat around 50 centimeters from the computer screen. The instructor provided them with full details concerning the aims of the study and the procedure to be followed, and they signed the written informed consent. Then, the participants filled in the questionnaires. Participants were then provided with the instructions of the ANT as well as with a training phase comprising 24 randomly selected trials. Participants then completed the ANT. We-Prime 2 Professional ${ }^{\circledR}$ to run the experiment. After the experiment, the participants were debriefed individually. HC participants received compensation (10 euros) for their participation but, to align with the ethical approval, in-patients from the MDD, GAD, and MDD/GAD groups did not receive compensation.

\subsection{Data Preparation and Analysis Plan}

\subsubsection{Power analysis.}

An a priori power analysis was computed to estimate the suitable total sample size for testing our hypothesis (G*Power 3.1.3; [62]). The power analysis indicated that a sample of at 
least 19 participants per group would yield adequate power (i.e., 90\%) to detect a small effect size of $f=.10$ at $p<.05$ using a 4-group MANOVA with the three attentional networks as dependent variables, thus confirming that this study has sufficient statistical power to test our hypothesis.

\subsubsection{Data preparation.}

As in previous research $[46,50,63]$, we first discarded data from trials with incorrect responses and RTs lower than $200 \mathrm{~ms}$ or greater than $2000 \mathrm{~ms}$ for each participant. Two subjects (one $\mathrm{MDD} / \mathrm{GAD}$ and one $\mathrm{HC}$ ) were excluded due to abnormaly elevated number of outliers and errors in their response pattern ${ }^{1}$. The four groups did not differ in terms of pourcentage of error rates, $F(3,117)=0.82, p=.48, \eta^{2} \mathrm{p}=.02\left[M_{\mathrm{MDD} / \mathrm{GAD}}=.01 \%, S D_{\mathrm{MDD} / \mathrm{GAD}}=.02 ; M_{\mathrm{MDD}}=\right.$ $\left..007 \%, S D_{\mathrm{MDD}}=.009 ; M_{\mathrm{GAD}}=.008 \%, S D_{\mathrm{GAD}}=.006 ; M_{\mathrm{HC}}=.006 \%, S D_{\mathrm{HC}}=0.006\right]$.

Following Fan et al. [37] and prior research on the use of ANT in mental health research [44-54], we computed the three attentional scores using the formulae depicted in Figure 1 (panel d): 1) the alerting effect was computed by subtracting the mean reaction time (RT) for double cue trials from the mean for no cue trials (No cue - Double cue); 2) the orienting effect by subtracting the mean for spatial cue trials from the mean result for center cue trials (Center cue - Spatial cue); 3) and the executive conflict effect by subtracting the mean for congruent trials (summed across cue types) from the mean for incongruent trials (Incongruent Congruent). For both alerting and orienting effects, greater subtraction scores for RT indicate greater efficiency. In contrast, greater subtraction scores for RT on executive conflict indicate increased difficulty with executive control of attention [37-39].

\subsubsection{Statistical Analyses.}

\footnotetext{
${ }^{1}$ Note that we analyzed the data with and without those participants. Their inclusion did not lead to any significant difference in the pattern of findings.
} 
We performed all statistical analyses using SPSS ${ }^{\circledR}$ version 25.0 (SPSS Inc.). Potential group differences in clinical severity and demographics were examined through separate oneway analyses of variance (ANOVAs). Given the multivariate nature of the dataset, the differences in the three attention networks were probed via a one-way multivariate analysis of covariance (MANCOVA), including the 3 attentional scores as dependent variables and the 4 groups as a fixed factor, while simultaneously controlling for education level (given the presence of a significant difference between groups in terms of education level ${ }^{2}$; see below $)^{3}$. For each analysis, the significance level was set at an alpha level of .05 (bilateral). Significant multivariate effects were followed-up with univariate ANCOVAs. Bonferroni-corrected $t$-tests were used to examine post hoc comparisons while controlling for multiple comparisons. Twotailed Pearson correlations (while controlling for education) were also computed to explore the relations between the three attentional scores (i.e., ANT scores) and GAD-7 and BDI-II scores). In an open science fashion, de-identified data as well as the data analysis code have been made publicly available via the Open Science Framework and can be accessed at https://osf.io/kjgn9/.

\section{Results}

\subsection{Groups description}

As shown in Table 1, the four groups did not significantly differ in age and gender. However, there was a significant difference in terms of years of education between groups.

Moreover, the clinical groups reported significantly higher levels of symptomatology (see Table 1). The GAD and MDD/GAD groups showed significantly higher scores on the GAD-7 than the MDD and HC groups, thus confirming the severity of the anxiety symptoms

\footnotetext{
${ }^{2}$ We also analyzed the data without controlling for education, and it resulted in the same patterns of findings. However, we decided to present the analyses with the covariate to be as conservative as possible.

${ }^{3}$ We only focused on the three indices, following prior research using the ANT in psychiatry [42-51]. Moreover, from a statistical perspective, examining and comparing each of the ANT trials' types among the four groups would have led to a linear model of 4 (Cue types) X 3 (Flanker types) X 4 (groups), with the two first factors in withinsubject and the last one in between-group design. In other words, it would have resulted in comparing 48 different cells between them (i.e., 2304 possible post-hoc comparisons). Such an approach would have obscured the interpretability of the present results, especially given our theory-driven approach grounded in a three-component framework of attention.
} 
within those two groups. In contrast, the MDD and MDD/GAD groups reported significantly higher scores on the BDI-II than the GAD and HC groups, thus endorsing the severity of the depression symptoms within those two groups.

\subsection{Difference in the attentional networks}

The MANCOVA revealed a significant multivariate main effect of Group, Wilks' $\lambda=$ $.77, F(9,270)=3.35, p<.01, \eta^{2} p^{=} .08$. The univariate ANCOVAs only showed a significant group difference for the executive control network, $F(4,117)=5.80, p<.001, \eta^{2} p^{=} .17$. There were no significant differences between groups for the alerting, $F(4,117)=.72, p=.58, \eta^{2} p^{=}$ .03 , or the orienting networks of attention, $F(4,117)=2.21, p=.07, \eta^{2} p^{=} .07$.

Follow-up Bonferroni-corrected $t$-tests revealed that the MDD/GAD group show significantly higher scores for the executive control network of attention, and thus higher impairments in the efficiency of this attentional network, than the GAD $(p=.006)$ and the $\operatorname{HC}$ $(p<.001)$ groups, but did not differ from the MDD group $(p=.24)$. Moreover, the GAD and MDD groups also exhibited significantly higher scores than the $\mathrm{HC}$ group ( $p=.03$ and $p=.001$, respectively) but were not significantly different from each other $(p=.10)$. Figure 2 shows the attentional network scores of each group.

\subsection{Associations between clinical severity and impairments in the attentional networks}

As depicted in Table 2, higher scores on either BDI or GAD-7 were associated with with more impairments of the executive control network of attention. None of the other correlations were significant.

\section{Discussion}


The main aim of this study was to compare, for the first time, the efficiency of the three attentional networks of patients with co-occurring GAD and MDD to patients with MDD solely, GAD solely, or free from GAD or MDD (i.e., healthy comparison group). Our findings reveal that the co-occurrence of MDD and GAD and either MDD or GAD solely are associated with specific impairments in the executive but not in the alerting or orienting networks of attention. Moreover, we found that patients with co-occurring MDD and GAD exhibited more severe impairments than those with MDD or GAD solely and that there was no significant difference between patients with MDD solely and GAD solely, though these latter groups show more significant impairment than those free from either diagnosis. Altogether, our findings thus call for a staging approach to the executive control's deficits, wherein patients with co-occurring disorders exhibit more impairment than those with only one disorder, and those latter showing more impairment than those free from any diagnosis.

Such a staging effect dovetails with longstanding health care models of comorbidity [64-71]. From this approach, a staging effect reflects a situation wherein the comorbidity between any pair of disorders (that is, physical and/or mental) is considered as more damaging than the impact of any one disorder alone ([64-67]; for mental health, see [68-71]). Note that this damaging effect has been especially viewed in terms of severity and functional impacts $[64,68]$. For instance, co-occurring anxiety and mood disorders have been associated with more distress and functional impairment in daily life than either of the disorders alone $[8,72,73]$. Here, we offered the very first observation that such a staging phenomenon may occur for executive control impairments in co-occurring MDD and GAD. Of note, we found that patients with co-occurring MDD and GAD did not perform across all conditions and that the staging effect was specific to the executive control network of attention. Should the patients with cooccurring MDD and GAD perform poorly across all the conditions, it would have led to 
significant differences between this group and the three others on all the ANT indices - an observation not confirmed in the present study.

To the best of our knowledge, this is also the first study of the field to include a group of patients with GAD solely. Indeed, in most previous studies, the very assessment of comorbid anxiety referred to distinct clinical phenomena, often departing from GAD. For instance, in some studies [30], comorbid anxiety denoted a high score on a scale assessing various anxiety features. In others, comorbid anxiety indicated the presence of at least one concurrent anxiety disorder-though often more than one comorbid disorder [29]. Altogether, this confusion regarding the construct of "comorbid anxiety" had blocked progress for years. Here, for the first time, we strictly aligned with the construct of GAD. Our findings thus also dovetail with previous theoretical and empirical works of top-down executive impairments in GAD patients compared to healthy comparison groups [54].

Of note, our observation of impaired executive control in GAD patients aligns with GAD's most prominent cognitive models $[17,74]$, which postulate that compromised top-down executive control may foster excessive worrying, once the hallmark symptom of GAD - a prediction confirmed by empirical research $[75,76]$. Like rumination, worrying has been designated a core transdiagnostic process of depression and anxiety-related psychopathology [77]. Therefore, a critical step for future research will be to examine the specific role of executive control in the co-occurrence between MDD and GAD and the potential contribution of both executive control and worrying. Beyond the issue of the comorbidity between MDD and GAD, many studies have emphasized the clinical importance of the interplay between executive control and repetitive negative thinking [78-80]. In particular, research has stressed the potential role of repetitive negative thinking in depleting top-down executive control resources, ultimately leading to a full-blown episode of GAD or MDD [28]. On the other hand, a couple of studies have suggested that it might also work the other way around, i.e., poor 
executive control triggering anxiety and depression via repetitive negative thinking [57]. A critical next step in future iterations will thus be to clarify the respective contributions of repetitive negative thinking and executive control in co-occurring MDD and GAD.

The present study may yield clinical implications. Prominent cognitive models of psychopathology have put forward attention control as a potential candidate for maintaining anxiety and depression [17,19-21,57]. Although our findings cannot directly speak to the maintenance of MDD, GAD, and their co-occurrence, they lend some support to mounting recommendations for the implementation of cognitive rehabilitation - that is, the use of psychological intervention targetting cognitive processes - in the alleviation of mental disorders (for a review, see [81]). Of clinical promise for practitioners, research already pointed to a few potent neuropsychological procedures for the rehabilitation of the attentional networks [82-84], and practitioners may thus capitalize on those targeting the executive control network [85-87]. Mindfulness training has also been identified as a highly promising and robust intervention for improving the executive control network [88,89]. Finally, recent studies have pointed to non-invasive brain stimulation techniques, and particularly transcranial direct current stimulation techniques, as plausible candidates to help restore the executive control network of attention in clinical and non-clinical samples $([90,91]$ but see [92]).

Several limitations require further examination in future iterations. First, our crosssectional study design precludes strong inference regarding the cause-effect relationship between executive control and the co-occurrence between MDD and GAD. Future studies may thus want to capitalize on longitudinal and experimental research methods to move us closer to elucidating these variables' relations. In this way, examining, through longitudinal research designs, whether impairments in the executive control network precedes the transition from MDD or GAD alone to their co-occurrence will be of critical importance in future research. 
Second, the symptoms' structure of co-occurring MDD and GAD may differ from those of patients with MDD or GAD only [69]. Notably, the association between each individual symptom and the three attentional scores may differ between our four groups. However, examining the symptom's organization and its association with each of the three attentional networks would have required a very different methodological approach-for instance, a network approach $[41,69]$. Interestedly, recent network research on the co-occurrence between MDD and GAD pointed to the critical role of executive control compared to the two other attentional networks in the connections with key symptoms common to MDD and GAD, and particularly sleep problems [93]. A critical step in future iteration would thus be to test whether this connection increases in patients with co-occurring MDD and GAD, as compared to those with MDD or GAD solely-i.e., in other words, whether one can expect a staging approach to the network structure of the relations between symptoms and attentional networks.

Third, in the group of patients with co-occurring MDD and GAD, we were unable to determine whether MDD preceded GAD or vice versa since we relied upon a DSM-5 structured interview, devoid of any considerations regarding the temporal order of the disorders. A critical step in future iterations would thus be to explore whether the results differ between patients with MDD with comorbid GAD and those with GAD with comorbid MDD. On the other hand, such an approach would require extensive longitudinal data collections. In the same vein, we focused on co-occurring GAD in MDD. A critical step would be to examine whether the present findings generalize to other anxiety and stress-related disorders that are frequently associated with MDD, like social anxiety disorder [94] or posttraumatic stress disorder [95].

Finally, patients with co-occurring MDD and GAD were significantly less educated. However, lower educational levels and poverty have unfortunately been associated with the severity of mental disorders - often reflected by their co-occurrence (for a review, see [96]). On the other hand, our conclusions remain unchanged when controlling for educational level. 


\section{Conclusions}

These limitations notwithstanding, this study is the first to examine the efficiency of the three attentional networks in patients with co-occurring GAD and MDD, as compared to patients with either of these disorders solely, thus filling a critical gap for the field's larger crusade to tackle comorbidity, one of the-if not the-toughest theoretical and clinical challenges of mental health research. 


\section{Declarations of interest}

Dr. Heeren receives honoraria for his editorial work from Elsevier (as Associate Editor of Journal of Anxiety Disorders). The authors declared that they had no other potential conflicts of interest with respect to their authorship or the publication of this article.

\section{Acknowledgement}

We are extremely thankful to M. Annelise Blanchard for her thoughtful comments on a previous version of this manuscript.

\section{Funding sources}

CC was supported by a research fellowship from the "Beau Vallon Psychiatric Hospital" (Grant "G01180016") and AH was supported by the F.R.S.-FNRS Belgian Science Foundation (Grant “1.C.059.18F”).

\section{Authors Contribution Statement}

$\mathrm{CC}$ and $\mathrm{AH}$ conceptualized the study. CC collected the data, analyzed the data, and drafted the manuscript. $\mathrm{CC}$ and $\mathrm{AH}$ contributed to the interpretation of data and to the drafting of the manuscript. AH and XDL revised the manuscript critically. All authors gave final approval of the current version to be published. 


\section{References}

[1] Brown TA, Campbell LA, Lehman CL, Grisham JR, Mancill RB. Current and lifetime comorbidity of the DSM-IV anxiety and mood disorders in a large clinical sample. J. Abnorm. Psychol. 2001;110(4):585-599. https://doi.org/10.1037/0021-843X.110.4.585

[2] Kessler RC, Chiu WT, Demler O, Walters EE. Prevalence, Severity, and Comorbidity of 12-Month DSM-IV Disorders in the National Comorbidity Survey Replication. Arch Gen Psychiatry 2005;62:617-627. https://doi.org/10.1001/archpsyc.62.6.617

[3] Ruscio AM, Hallion LS, Lim CCW, et al. Cross-sectional Comparison of the Epidemiology of DSM-5 Generalized Anxiety Disorder Across the Globe. JAMA Psychiatry. 2017;74(5):465. https://doi.org/10.1001/jamapsychiatry.2017.0056

[4] Olfson M, Mojtabai R, Merikangas KR, et al. Reexamining associations between mania, depression, anxiety and substance use disorders: results from a prospective national cohort. Mol Psychiatry. 2017;22(2):235-241. https://doi.org/10.1038/mp.2016.64

[5] Kessler RC, Stang PE, Wittchen HU, Ustun TB, Roy-Burne PP, Walters EE. Lifetime panic-depression comorbidity in the National Comorbidity Survey. Arch Gen Psychiatry. 1998;55(9):801-808. https://doi.org/10.1001/archpsyc.55.9.801

[6] Hirschfeld RMA. The Comorbidity of Major Depression and Anxiety Disorders: Recognition and Management in Primary Care. Prim Care Companion J Clin Psychiatry. 2001;3(6):244-254. https://doi.org/10.4088/pcc.v03n0609

[7] Dold M, Bartova L, Souery D, et al. Clinical characteristics and treatment outcomes of patients with major depressive disorder and comorbid anxiety disorders - results from a European multicenter study. J. Psychiat. Res 2017;91:1-13. https://doi.org/10.1016/j.jpsychires.2017.02.020

[8] Schoevers RA, Deeg DJH, van Tilburg W, Beekman ATF. Depression and Generalized Anxiety Disorder: Co-Occurrence and Longitudinal Patterns in Elderly Patients. Am. J. Geriatr. Psychiatry 2005;13(1):31-39. https://doi.org/10.1097/00019442-20050100000006

[9] Zhou Y, Cao Z, Yang M, et al. Comorbid generalized anxiety disorder and its association with quality of life in patients with major depressive disorder. Sci Rep. 2017;7. https://doi.org/10.1038/srep40511

[10] Niles AN, Dour HJ, Stanton AL, et al. Anxiety and depressive symptoms and medical illness among adults with anxiety disorders. J. Psychosom. Res. 2015;78(2):109-115. https://doi.org/10.1016/j.jpsychores.2014.11.018

[11] Nock M, Hwang I, Sampson N, Kessler R. Mental Disorders, Comorbidity and Suicidal Behavior: Results from the National Comorbidity Survey Replication. Mol Psychiatry. 2010;15(8):868-876. https://doi.org/10.1038/mp.2009.29

[12] Ballenger JC. Anxiety and Depression: Optimizing Treatments. Prim Care Companion J Clin Psychiatry. 2000;2(3):71-79. https://doi.org/10.4088/pcc.v02n0301 
[13] Coplan JD, Aaronson CJ, Panthangi V, Kim Y. Treating comorbid anxiety and depression: Psychosocial and pharmacological approaches. World J Psychiatry. 2015;5(4):366-378. https://doi.org/10.5498/wjp.v5.i4.366

[14] Saveanu R, Etkin A, Duchemin A-M, et al. The international Study to Predict Optimized Treatment in Depression (iSPOT-D): outcomes from the acute phase of antidepressant treatment. J Psychiatr Res. 2015;61:1-12. https://doi.org/10.1016/j.jpsychires.2014.12.018

[15] Brown C, Schulberg HC, Madonia MJ, Shear MK, Houck PR. Treatment outcomes for primary care patients with major depression and lifetime anxiety disorders. Am J Psychiatry. 1996;153(10):1293-1300. https://doi.org/10.1176/ajp.153.10.1293

[16] van Balkom AJLM, van Boeijen CA, Boeke AJP, van Oppen P, Kempe PT, van Dyck R. Comorbid depression, but not comorbid anxiety disorders, predicts poor outcome in anxiety disorders. Depress Anxiety. 2008;25(5):408-415. https://doi.org/10.1002/da.20386

[17] Eysenck MW, Derakshan N. New perspectives in attentional control theory. Pers. Individ. Differ 2011;50(7):955-960. https://doi.org/10.1016/j.paid.2010.08.019

[18] Heeren A, De Raedt R, Koster EHW, Philippot P. The (neuro)cognitive mechanisms behind attention bias modification in anxiety: proposals based on theoretical accounts of $\begin{array}{llll}\text { attentional bias. Hum } & \text { Front }\end{array}$ https://doi.org/10.3389/fnhum.2013.00119

[19] Hammar Å, Årdal G. Cognitive Functioning in Major Depression - A Summary. Front Hum Neurosci. 2009;3:26. https://doi.org/10.3389/neuro.09.026.2009

[20] Keller AS, Leikauf JE, Holt-Gosselin B, Staveland BR, Williams LM. Paying attention to attention in depression. Transl. Psychiatry. 2019;9(1):1-12. https://doi.org/10.1038/s41398-019-0616-1

[21] Koster EHW, De Lissnyder E, Derakshan N, De Raedt R. Understanding depressive rumination from a cognitive science perspective: the impaired disengagement hypothesis. Clin Psychol Rev. 2011;31(1):138-145. https://doi.org/10.1016/j.cpr.2010.08.005

[22] Cuthbert BN, Insel TR. Toward the future of psychiatric diagnosis: the seven pillars of RDoC. BMC medicine. 2013;11:126. https://doi.org/10.1186/1741-7015-11-126

[23] Abramovitch A, Short T, Schweiger A. The c factor: Cognitive dysfunction as a transdiagnostic dimension in psychopathology. Clin Psychol Review. 2021:102007. https://doi.org/10.1016/j.cpr.2021.102007

[24] Hilgard ER. The trilogy of mind: Cognition, affection, and conation. Journal of the J Hist. Behav. Sci. 1980;16(2):107-117.

[25] Hallion LS, Tolin DF, Assaf M, Goethe J, Diefenbach GJ. Cognitive Control in Generalized Anxiety Disorder: Relation of Inhibition Impairments to Worry and Anxiety Severity. Cogn Ther Res. 2017;41(4):610-618. https://doi.org/10.1007/s10608-017-9832$\underline{2}$ 
[26] Porter RJ, Bourke C, Gallagher P. Neuropsychological impairment in major depression: its nature, origin and clinical significance. Aust N Z J Psychiatry. 2007;41(2):115-128. https://doi.org/10.1080/00048670601109881

[27] Snyder HR. Major Depressive Disorder Is Associated with Broad Impairments on Neuropsychological Measures of Executive Function: A Meta-Analysis and Review. Psychol. Bul. 2013;139(1):81-132.

[28] Stefanopoulou E, Hirsch CR, Hayes S, Adlam A, Coker S. Are attentional control resources reduced by worry in generalized anxiety disorder? J Abnorm Psychol. 2014;123(2):330-335. https://doi.org/10.1037/a0036343

[29] Basso MR, Lowery N, Ghormley C, et al. Comorbid anxiety corresponds with neuropsychological dysfunction in unipolar depression. Cogn Neuropsychiatry. 2007;12(5):437-456. https://doi.org/10.1080/13546800701446517

[30] Lyche P, Jonassen R, Stiles TC, Ulleberg P, Landro NI. Attentional Functions in Major Depressive Disorders With and Without Comorbid Anxiety. Arch Clin Neuropsychol. 2011;26(1):38-47. https://doi.org/10.1093/arclin/acq095

[31] Castaneda AE, Marttunen M, Suvisaari J, et al. The effect of psychiatric co-morbidity on cognitive functioning in a population-based sample of depressed young adults. Psychol Med. 2010;40(1):29-39. https://doi.org/10.1017/S0033291709005959

[32] Herrera-Guzmán I, Gudayol-Ferré E, Jarne-Esparcia A, et al. Comorbidity of anxiety disorders in major depressive disorder: A clinical trial to evaluate neuropsychological deficit. Eur. Psychiatry. 2009;23(1):5-18.

[33] Shi R, Sharpe L, Abbott M. A meta-analysis of the relationship between anxiety and attentional control. Clin Psychol Rev. 2019;72:101754. https://doi.org/10.1016/j.cpr.2019.101754

[34] Heeren A, Maurage P, Philippot P. Revisiting attentional processing of non-emotional cues in social anxiety: A specific impairment for the orienting network of attention. Psychiatry Res. 2015;228(1):136-142. https://doi.org/10.1016/j.psychres.2015.04.030

[35] Moriya J, Tanno Y. Dysfunction of attentional networks for non-emotional processing in negative affect. Cogn. Emot. 2009;23(6):1090-1105. https://doi.org/10.1080/02699930802335018

[36] Eronen MI, Bringmann LF. The Theory Crisis in Psychology: How to Move Forward. Perspect Psychol Sci. 2021:1745691620970586. https://doi.org/10.1177/1745691620970586

[37] Fan J, McCandliss BD, Sommer T, Raz A, Posner MI. Testing the efficiency and independence of attentional networks. J Cogn Neurosci. 2002;14(3):340-347. https://doi.org/10.1162/089892902317361886

[38] Petersen SE, Posner MI. The Attention System of the Human Brain: 20 Years After. Annu Rev Neurosci. 2012;35:73-89. https://doi.org/10.1146/annurev-neuro-062111-150525 
[39] Posner MI, Rothbart MK. Research on Attention Networks as a Model for the Integration of Psychological Science. Annu. Rev. Clin. Psychol. 2007;58(1):1-23. https://doi.org/10.1146/annurev.psych.58.110405.085516

[40] Raz A, Buhle J. Typologies of attentional networks. Nat. Rev. Neurosci. 2006;7:367-379. https://doi.org/10.1038/nrn1903

[41] Heeren A, Hoebeke Y, Coussement C. Unfolding the Complex Dynamic Interplay Between Attentional Processes and Anxiety: A Commentary on Ghassemzadeh, Rothbart, and Posner. Cogn. Behav. Neurol. $2019 ; 32(1)$ : 63-66. https://doi.org/10.1097/WNN.0000000000000187

[42] Posner MI. Orienting of attention. Q J Exp Psychol. 1980;32(1):3-25. https://doi.org/10.1080/00335558008248231

[43] Eriksen BA, Eriksen CW. Effects of noise letters upon the identification of a target letter in a nonsearch task. Percept and Psychophys 1974;16(1):143-149. https://doi.org/10.3758/BF03203267

[44] Gooding DC, Braun JG, Studer JA. Attentional network task performance in patients with schizophrenia-spectrum disorders: Evidence of a specific deficit. Schizophr. Res. 2006;88(1-3):169-178. https://doi.org/10.1016/j.schres.2006.07.009

[45] Lannoy S, Heeren A, Moyaerts N, et al. Differential impairments across attentional networks in binge drinking. Psychopharmacology (Berl). 2017;234(7):1059-1068. https://doi.org/10.1007/s00213-017-4538-4

[46] Maurage P, de Timary P, Billieux J, Collignon M, Heeren A. Attentional Alterations in Alcohol Dependence Are Underpinned by Specific Executive Control Deficits. Alcohol: Clin. Exp. Res. 2014;38(7):2105-2112. https://doi.org/10.1111/acer.12444

[47] Mullane JC, Corkum PV, Klein RM, McLaughlin EN, Lawrence MA. Alerting, orienting, and executive attention in children with ADHD. J Atten Disord. 2011;15(4):310-320. https://doi.org/10.1177/

[48] Urbanek C, Weinges-Evers N, Bellmann-Strobl J, et al. Attention Network Test reveals alerting network dysfunction in multiple sclerosis. Mult Scler. 2010;16(1):93-99. https://doi.org/10.1177/1352458509350308

[49] Wang H, Mo C, Fang F. Dissociated deficits in attentional networks in social anxiety and depression. Sci China Life Sci. 2020;63:1071-1078. https://doi.org/10.1007/s11427-019$1624-5$

[50] Heeren A, Maurage P, Perrot H, et al. Tinnitus specifically alters the top-down executive control sub-component of attention: Evidence from the Attention Network Task. Behav. Brain Res. 2014;269:147-154. https://doi.org/10.1016/j.bbr.2014.04.043

[51] Maurage P, Heeren A, Lahaye M, et al. Attentional impairments in Huntington's disease: A specific deficit for the executive conflict. Neuropsychology. 2017;31(4):424-436. https://doi.org/10.1037/neu0000321 
[52] Togo F, Lange G, Natelson BH, Quigley KS. Attention network test: Assessment of cognitive function in chronic fatigue syndrome. J Neuropsychol 2015;9(1):1-9. https://doi.org/10.1111/jnp.12030

[53] Heeren A, McNally RJ. An integrative network approach to social anxiety disorder: The complex dynamic interplay among attentional bias for threat, attentional control, and symptoms. J. Anxiety Disord 2016;42:95-104. https://doi.org/10.1016/j.janxdis.2016.06.009

[54] Pacheco-Unguetti AP, Acosta A, Marqués E, Lupiáñez J. Alterations of the attentional networks in patients with anxiety disorders. J. Anxiety Disord 2011;25(7):888-895. https://doi.org/10.1016/j.janxdis.2011.04.010

[55] Gruber S a, Rathgeber K b, Bräunig P b, Gauggel S a. Stability and course of neuropsychological deficits in manic and depressed bipolar patients compared to patients with Major Depression. J Affect Disord. 2007;104(1-3):61-71. https://doi.org/10.1016/j.jad.2007.02.011

[56] Ossola P, Ferrari M, Lucarini V, et al. Role of the attentional networks in the psychopathology of bipolar disorder. Eur. Neuropsychopharmacology. 2018;28:S73-S74. https://doi.org/10.1016/j.euroneuro.2017.12.106

[57] Hsu KJ, Beard C, Rifkin L, Dillon DG, Pizzagalli DA, Björgvinsson T. Transdiagnostic mechanisms in depression and anxiety: The role of rumination and attentional control. $\mathrm{J}$ Affect Disord 2015;188:22-27. https://doi.org/10.1016/j.jad.2015.08.008

[58] Zimmerman M. Interview Guide for Evaluating DSM-5 Psychiatric Disorders and the Mental Status Examination. Psych Products Press; 2013.

[59] Beck AT, Steer RA, Brown GK. Inventaire de Dépression de Beck - 2ème Édition (IDBII) [Beck Depression Inventory - 2nd Edition (BDI-II)]. Editions du Centre de Psychologie Appliquée.; 1998.

[60] Spitzer RL, Kroenke K, Williams JBW, Löwe B. A brief measure for assessing generalized anxiety disorder: the GAD-7. Arch Intern Med. 2006;166(10):1092-1097. https://doi.org/10.1001/archinte.166.10.1092

[61] Micoulaud-Franchi J-A, Lagarde S, Barkate G, et al. Rapid detection of generalized anxiety disorder and major depression in epilepsy: Validation of the GAD-7 as a complementary tool to the NDDI-E in a French sample. Epilepsy Behav. 2016;57(Pt A):211-216. https://doi.org/10.1016/j.yebeh.2016.02.015

[62] Faul F, Erdfelder E, Lang A-G, Buchner A. G*Power 3: a flexible statistical power analysis program for the social, behavioral, and biomedical sciences. Behav Res Methods. 2007;39(2):175-191. https://doi.org/10.3758/BF03193146

[63] Coussement C, Riesco de Vega M, Heeren A. The impact of anodal tDCS on attentional networks as a function of trait anxiety and depressive symptoms: A preregistered doubleblind sham-controlled experiment. Clin Neuropsychiatry. 2020;17:225-235. https://doi.org/10.36131/cnfioritieditore20200404 
[64] Addington J, Liu L, Goldstein BI, et al. Clinical staging for youth at-risk for serious mental illness. Early Interv Psychiatry 2019;13(6):1416-1423. https://doi.org/10.1111/eip.12786

[65] Atlantis E, Sahebolamri M, Cheema BS, Williams K. Usefulness of the Edmonton Obesity Staging System for stratifying the presence and severity of weight-related health problems in clinical and community settings: A rapid review of observational studies. Obes. Rev 2020;21(11):e13120. https://doi.org/10.1111/obr.13120

[66] Feinstein AR, Wells CK. A Clinical-Severity Staging System for Patients with Lung Cancer. Medicine. 1990;69(1):1-33. https://doi.org/10.1097/00005792-199001000-00001

[67] Ndetei D, Musyimi C, Tele A, Musau A, Gitonga I, Mutiso V. Psychological Well-Being and Social Functioning Across the Cancer Stages: Implications for Palliative Care. J Soc Work End Life Palliat Care 2018;14(2-3):194-208. 10.1080/15524256.2018.1472718

[68] Bokma WA, Batelaan NM, Hoogendoorn AW, Penninx BW, van Balkom AJ. A clinical staging approach to improving diagnostics in anxiety disorders: Is it the way to go? Aust N Z J Psychiatry. 2020;54(2):173-184. 10.1177/0004867419887804

[69] Cramer AOJ, Waldorp LJ, van der Maas HLJ, Borsboom D. Comorbidity: A network perspective. Behav Brain Sci 2010;33(2-3):137-150. https://doi.org/10.1017/S0140525X09991567

[70] Kleiman EM, Riskind JH. Cognitive vulnerability to comorbidity: Looming cognitive style and depressive cognitive style as synergistic predictors of anxiety and depression symptoms. J. Behav. Ther. Exp. Psychiatry. 2012;43(4):1109-1114. https://doi.org/10.1016/j.jbtep.2012.05.008

[71] Macdonald AN, Goines KB, Novacek DM, Walker EF. Prefrontal mechanisms of comorbidity from a transdiagnostic and ontogenic perspective. Dev Psychopathol. 2016;28(4pt1):1147-1175. https://doi.org/10.1017/S0954579416000742

[72] Albert U, Rosso G, Maina G, Bogetto F. Impact of anxiety disorder comorbidity on quality of life in euthymic bipolar disorder patients: differences between bipolar I and II subtypes. J Affect Disord. 2008;105(1-3):297-303. https://doi.org/10.1016/j.jad.2007.05.020

[73] Brown TA, Antony MM, Barlow DH. Diagnostic comorbidity in panic disorder: Effect on treatment outcome and course of comorbid diagnoses following treatment. J. Consult. Clin. Psychol 1995;63(3):408-418. https://doi.org/10.1037/0022-006X.63.3.408

[74] Hirsch CR, Mathews A. A cognitive model of pathological worry. Behav. Res. Ther 2012;50(10):636-646. https://doi.org/10.1016/j.brat.2012.06.007

[75] Hallion LS, Steinman SA, Kusmierski SN. Difficulty Concentrating in Generalized Anxiety Disorder: An Evaluation of Incremental Utility and Relationship to Worry. J Anxiety Disord. 2018;53:39-45. https://doi.org/10.1016/j.janxdis.2017.10.007

[76] Zainal NH, Newman MG. Executive function and other cognitive deficits are distal risk factors of generalized anxiety disorder 9 years later. Psychol Med. 2018;48(12):20452053. https://doi.org/10.1017/S0033291717003579 
[77] McEvoy PM, Salmon K, Hyett MP, et al. Repetitive Negative Thinking as a Transdiagnostic Predictor of Depression and Anxiety Symptoms in Adolescents. Assessment. 2019;26(2):324-335. https://doi.org/10.1177/1073191117693923

[78] Bernstein EE, Heeren A, McNally RJ. Unpacking Rumination and Executive Control: A Network Perspective. Clin. Psychol. Sci. 2017;5(5):816-826. https://doi.org/10.1177/2167702617702717

[79] Philippot P, Agrigoroaei S. Repetitive thinking, executive functioning, and depressive mood in the elderly. Aging Ment. Health 2017;21(11):1192-1196. https://doi.org/10.1080/13607863.2016.1211619

[80] Zetsche U, Bürkner P-C, Schulze L. Shedding light on the association between repetitive negative thinking and deficits in cognitive control - A meta-analysis. Clin Psychol Rev. 2018;63:56-65. https://doi.org/10.1016/j.cpr.2018.06.001

[81] Kim EJ, Bahk Y-C, Oh H, Lee W-H, Lee J-S, Choi K-H. Current Status of Cognitive Remediation for Psychiatric Disorders: A Review. Front Psychiatry. 2018;9. https://doi.org/10.3389/fpsyt.2018.00461

[82] Robertson IH. Cognitive rehabilitation: attention and neglect. Trends Cogn Sci. 1999;3(10):385-393. https://doi.org/10.1016/s1364-6613(99)01378-9

[83] Robertson IH, Tegnér R, Tham K, Lo A, Nimmo-Smith I. Sustained attention training for unilateral neglect: theoretical and rehabilitation implications. J Clin Exp Neuropsychol. 1995;17(3):416-430. https://doi.org/10.1080/01688639508405133

[84] Thimm M, Fink GR, Küst J, Karbe H, Sturm W. Impact of alertness training on spatial neglect: a behavioural and fMRI study. Neuropsychologia. 2006;44(7):1230-1246. https://doi.org/10.1016/j.neuropsychologia.2005.09.008

[85] Cicerone KD, Maestas KL. Rehabilitation of Attention and Executive Function Impairments. In: Sherer M, Sander AM, eds. Handbook on the Neuropsychology of Traumatic Brain Injury. Clinical Handbooks in Neuropsychology. Springer; 2014:191211. https://doi.org/10.1007/978-1-4939-0784-7_10

[86] Serino A, Ciaramelli E, Santantonio AD, Malagù S, Servadei F, Làdavas E. A pilot study for rehabilitation of central executive deficits after traumatic brain injury. Brain Inj. 2007;21(1):11-19. https://doi.org/10.1080/02699050601151811

[87] Sturm W, Willmes K, Orgass B, Hartje W. Do specific attention deficits need specific training? Neuropsychol Rehabi 1997;7(2):81-103. https://doi.org/10.1080/713755526

[88] Tang Y-Y, Ma Y, Wang J, et al. Short-term meditation training improves attention and self-regulation. Proc Natl Acad Sci U S A. 2007;104(43):17152-17156. https://doi.org/10.1073/pnas.0707678104

[89] Heeren A, Van Broeck N, Philippot P. The effects of mindfulness on executive processes and autobiographical memory specificity. Behav Res Ther. 2009;47(5):403-409. https://doi.org/10.1016/j.brat.2009.01.017 
[90] Miler JA, Meron D, Baldwin DS, Garner M. The Effect of Prefrontal Transcranial Direct Current Stimulation on Attention Network Function in Healthy Volunteers: tDCS and Attention Network Function. Neuromodulation. 2017;21(4):355-361. https://doi.org/10.1111/ner.12629

[91] Silva AF, Zortea M, Carvalho S, et al. Anodal transcranial direct current stimulation over the left dorsolateral prefrontal cortex modulates attention and pain in fibromyalgia: randomized clinical trial. Sci Rep. 2017;7(1):135. https://doi.org/10.1038/s41598-017$\underline{00185-\mathrm{w}}$

[92] Coussement C, Maurage P, Billieux J, Heeren A. Does Change in Attention Control Mediate the Impact of tDCS on Attentional Bias for Threat? Limited Evidence from a Double-blind Sham-controlled Experiment in an Unselected Sample. Psychol Belg. 2019;59(1):16-32. https://doi.org/10.5334/pb.449

[93] Coussement C, Heeren A. Sleep problems as a transdiagnostic hub bridging impaired attention control, generalized anxiety, and depression. J Affect Disord. 2022;296:305-308. https://doi.org/10.1016/j.jad.2021.09.092

[94] Heeren A, Jones PJ, McNally RJ. Mapping network connectivity among symptoms of social anxiety and comorbid depression in people with social anxiety disorder. J Affect Disord. 2018;228:75-82. https://doi.org/10.1016/j.jad.2017.12.003

[95] Lazarov A, Suarez-Jimenez B, Levi O, et al. Symptom structure of PTSD and co-morbid depressive symptoms - a network analysis of combat veteran patients [published correction appears in Psychol Med. 2020 Oct;50(13):2171]. Psychol Med. 2020;50(13):2154-2170. https://doi.org/10.1017/S0033291719002034

[96] Ridley M, Rao G, Schilbach F, Patel V. Poverty, depression, and anxiety: Causal evidence and mechanisms. Science. 2020;370(6522). https://doi.org/10.1126/science.aay0214 


\section{FIGURE CAPTIONS}

\section{Figure 1. Illustration of the Attention Network Task (ANT)}

Note. (a) example of a trial sequence; (b) the four possible cues; (c) the six possible targets; (d) the scores computation. Adapted from Fan et al. [37].

Figure 2. Comparison of the three attentional networks reaction time in the four groups controlling for education level.

Note. Error bars show the standard error of the mean. Covariate appearing in the model is evaluated as an education level $=14.22$. GAD $=$ Patients with GAD, MDD $=$ Patients with $\mathrm{MDD}, \mathrm{MDD} / \mathrm{GAD}=$ Patients with co-occurring MDD and GAD.

$* p<.05 ; * * p<.01 ; * * * p<.001$ 

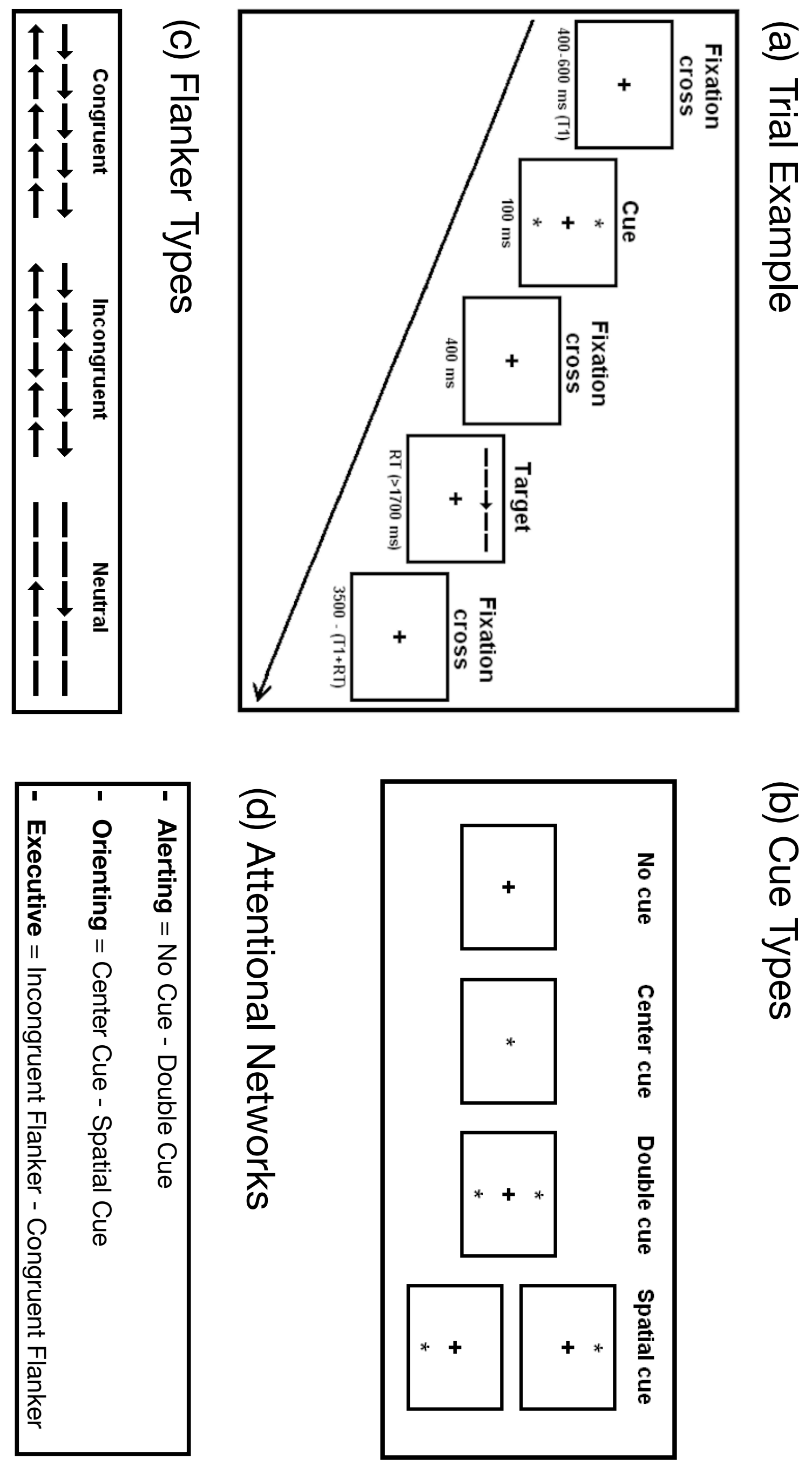


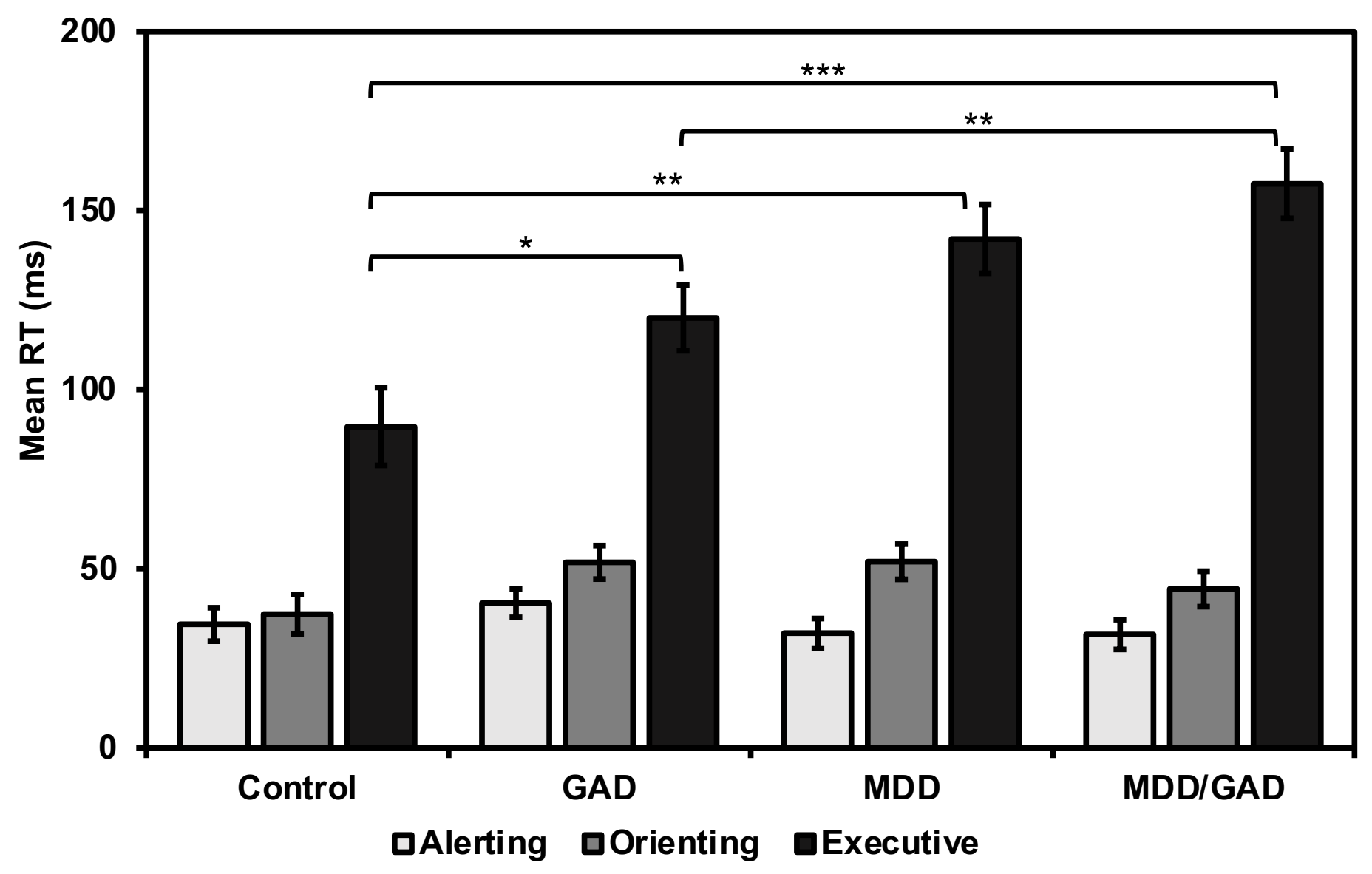


Table 1. Participant's characteristics (SD in parentheses).

\begin{tabular}{lccccc}
\hline & $\begin{array}{c}\text { Control } \\
(n=29)\end{array}$ & $\begin{array}{c}\text { GAD } \\
(n=30)\end{array}$ & $\begin{array}{c}\text { MDD } \\
(n=30)\end{array}$ & $\begin{array}{c}\text { GAD/MDD } \\
(n=29)\end{array}$ & $F$ or $\chi^{2}$ \\
\hline Age & $29.34(8.35)$ & $29.40(11.61)$ & $35.73(14.99)$ & $32.97(7.43)$ & $2.31^{\mathrm{a}}$ \\
\%Female & 75.1 & 80 & 53.3 & 79.3 & $7.12^{\mathrm{b}}$ \\
Education & $17.41(2.33)$ & $14.23(3.21)$ & $12.57(2.45)$ & $12.72(2.67)$ & $20.32^{\mathrm{a}^{*}}$ \\
BDI-II & $4.93(4.44)$ & $13.13(4.21)$ & $26.40(7.60)$ & $33.66(12.60)$ & $77.16^{\mathrm{a}^{* *}}$ \\
GAD-7 & $2.14(1.97)$ & $12.23(3.85)$ & $8.53(5.38)$ & $14.28(4.56)$ & $48.00^{\mathrm{a}^{* *}}$
\end{tabular}

Note. GAD = Generalized Anxiety Disorder; MDD = Major Depressive Disorder; GAD/MDD $=$ Co-occurring Generalized Anxiety Disorder and Major Depressive Disorder. Education level was assessed according to the number of years of education completed since starting primary school. BDI-II, Beck Depression Inventory ( $2^{\text {nd }}$ edition); GAD-7, Generalized Anxiety Disorder 7-items.

${ }^{\text {a }}$ Value for $F(3,114)$

${ }^{\mathrm{b}}$ Value for $\chi^{2}(3, \mathrm{~N}=118)$

$* p<0.05 ; * * p<0.01$ 
Table 2. Pearson correlations between the three attentional networks and clinical severity.

Alerting Orienting $\quad$ Executive Control

$\begin{array}{lrrl}\text { BDI-II } & -.05 & -.06 & \mathbf{3 6}^{* *} \\ \text { GAD-7 } & .04 & -.01 & \mathbf{3 8}^{* *}\end{array}$

Note. These correlations include the level of education as a covariate. BDI-II $=$ Beck Depression Inventory ( $2^{\text {nd }}$ edition $)$, GAD-7 $=$ Generalized Anxiety Disorder Scale. $* * p<0.01$ 